\title{
The Earliest Tau Dysfunction in Alzheimer's Disease?
}

\section{Tau Phosphorylated at S422 as a Toxic Seed}

\author{
Kellen Voss, John Koren III, and Chad A. Dickey \\ From the USF Health Byrd Alzheimer's Institute, Department of \\ Molecular Medicine, University of South Florida, Tampa, Florida
}

Despite being first described in 1907, the causative mechanism driving progression of Alzheimer's disease (AD) from normal healthy neurons to a diseased state remains to be elucidated. There are two major pathological markers that are associated with AD diagnosis: amyloid $\beta(A \beta)$ plaques and neurofibrillary tangles (NFTs). These extracellular plaques are composed of $A \beta 42$, a proteolytically cleaved product from the larger amyloid precursor protein. The intracellular NFTs are primarily composed of the hyperphosphorylated microtubule-associated tau protein. Despite both markers being required for an AD diagnosis, only NFT formation has a positive correlation with the onset and severity of dementia. ${ }^{1,2}$ The progression of tau pathological characteristics in $A D$ is known to originate from the transentorhinal cortex and progress through the hippocampus and into the neocortex. $^{2,3}$ Tau pathological characteristics have also been observed in cholinergic basal forebrain (CBF) neurons early in AD (Braak stages I, II, and III). ${ }^{4-6}$ These cholinergic neurons innervate the areas of the originating tau pathological characteristics. The progression of NFT formation during $A D$ in these cholinergic neurons is unknown. The precise mechanism of NFT formation still remains unknown; however, it is believed to begin with hyperphosphorylation of tau, resulting in a reduced affinity for microtubules. This free tau forms oligomeric structures that elongate into filaments and eventually coalesce into the larger aggregates, such as NFTs.

\section{pS422 Provides a Clue}

In this issue of The American Journal of Pathology, Vana et $\mathrm{al}^{7}$ sought to determine how progression of tau pathological characteristics is changed from normal aged brains to pathological AD brains in the CBF. They showed that pretangle formation in the $\mathrm{CBF}$ is associated with cognitive changes before NFT formation. This was achieved by analyzing brain sections of patients diagnosed as having no cognitive impairment, mild cognitive impairment, or $A D$, stained for biomarkers of $A D$ progression. One of these biomarkers was tau phosphorylated on S422 (pS422). This post-translational modification is recognized by a site-specific antibody that correlates with early disease events. ${ }^{8}$ Other biomarkers were used to examine events associated with later-stage $A D$, such as caspasemediated proteolytic cleavage of tau at aspartic acid 421 , by using tau antibody TauC3., ${ }^{9,10}$ To localize and monitor degeneration of the cholinergic neurons, Vana et $\mathrm{al}^{7}{ }^{7}$ used a marker for nerve growth factor receptor (p75 NTR). p75 ${ }^{\text {NTR }}$ binds nerve growth factor, a vital substance for CBF survival; in the absence of the $p 75^{\mathrm{NTR}}$, the CBF neurons undergo degeneration. ${ }^{11}$

S422 has recently gained notoriety owing to its involvement in NFT formation on phosphorylation in both in vitro and in vivo experiments. ${ }^{12-15}$ Other results indicate that, although $A \beta$ may not be correlative with degeneration, there is a link between fibrillization of $A \beta 42$ and the induction of phosphorylation at S422 in the amygdala. ${ }^{12}$ Similarly, in a pro-aggregation (P301L) model of SY5Y cells, $A \beta 42$ induction increased insoluble tau and paired helical filament-like filaments with P301L and P301L/ S422E tau, a pseudophosphorylated form, but not P301L/ S422A tau, an unphosphorylated form, of tau at S422. ${ }^{16}$

Intriguingly, recent work ${ }^{17}$ suggests that tau S422 is phosphorylated by a member of the mitogen-activated protein kinase (MAPK) superfamily, MAPK kinase 4. In addition to directly phosphorylating tau, MAPK kinase 4 also activates other MAPKs, and the downstream consequences of

Supported by NIH (R01NS073899 and R00AG031291), Alzheimer's Association (IIRG-09-130689 and NIRG-10-174517), Rosalinde and Arthur Gilbert New Investigator Awards in Alzheimer's disease/American Federation for Aging Research, and Irene and Abe Pollin Fund for Corticobasal Degeneration Research/Cure PSP.

Accepted for publication August 22, 2011.

CME Disclosure: None of the authors disclosed any relevant financial relationships.

Address reprint requests to Chad A. Dickey, Ph.D., Department of Molecular Medicine, University of South Florida, 12901 Bruce B. Downs Blvd. MDC 8, Tampa, FL 33612. E-mail: cdickey1@health.usf.edu. 
these other MAPKs can be vast. Other MAPKs can directly phosphorylate tau at distinct sites, ${ }^{18-21}$ and they can also regulate synaptic plasticity, excitotoxicity, cell-cycle re-entry, and oxidative stress. ${ }^{22}$ Each of these events has been linked to NFT formation and $A \beta$ deposition. ${ }^{23}$ Thus, phosphorylation of tau at $\mathrm{S} 422$ could be one of the earliest signs that the MAPK pathways are becoming dysregulated in AD, an outcome that could lead to a host of devastating problems for the brain.

\section{Proteolytic Cleavage of Tau May Spare Neurons}

Vana et $\mathrm{al}^{7}$ also observed a significant correlation between the progression of disease from no cognitive impairment to mild cognitive impairment and $A D$, with increases in pS422 levels and decreases in p75 ${ }^{\mathrm{NTR}+}$ cholinergic neurons; however, the number of neurons immunoreactive for both pS422 and p75 ${ }^{\mathrm{NTR}}$ progressively increased with Braak staging. More important, pS422 levels in CBF neurons significantly correlated with AD progression, but TauC3 levels, at least initially, did not, consistent with previous findings ${ }^{8}$ showing that phosphorylation at S422 can block caspase cleavage of tau. This finding also correlates well with data showing that neurons with caspase-mediated proteolytically cleaved tau (as indicated by TauC3 staining) actually live longer than those without TauC3 immunoreactivity. ${ }^{24}$ In this way, caspase-mediated cleavage of tau may actually be a neuroprotective response to the aberrant accumulation of tau. ${ }^{24}$ These results are also in agreement with previous results in a P301L pR5 mouse model, in which the phosphorylation of tau during pretangle formation (not the NFT number) had a greater impact on cognitive decline and neurodegeneration. ${ }^{14}$

\section{The Two Pools of Tau: Accumulation Impairs Clearance}

Perhaps the most intriguing aspect of this study is that as AD progressed, TauC3 immunoreactivity eventually emerged in the CBF neurons. At first, this may seem contradictory to previous data ${ }^{8}$ showing that phosphorylation at the S422 site prevents cleavage of tau by caspase 3 at D421. Recent data ${ }^{25}$ may shed light, however, on this seemingly surprising result. Indeed, caspase-mediated cleavage of tau must occur for its sequestration to the autophagy pathway. Without this proteolytic cleavage event, tau is not cleared by the workhorse pathway of the cell and may overburden the more specialized proteasome pathway, or even actively suppress it, facilitating accumulation of otherwise normal tau species (Figure 1). Thus, MAPK kinase 4 may precipitate tau dysfunction by producing a toxic seed incapable of clearance by autophagy: Phosphorylation at S422 prevents normal autophagic clearance of tau, which, in turn, leads to its accumulation and subsequent suppression of other clearance pathways. As a result, normal tau may start to accumulate secondarily. Thus, two distinct pools of tau may accumulate: one that is phosphorylated at S422 and cannot be proteolytically cleaved by

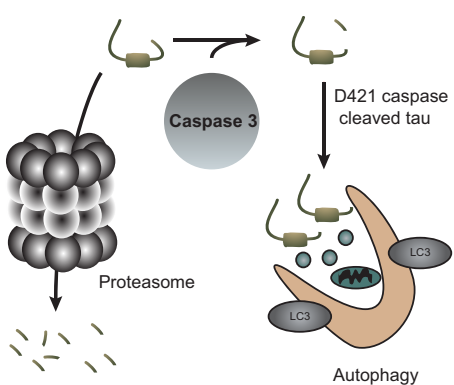

Normal

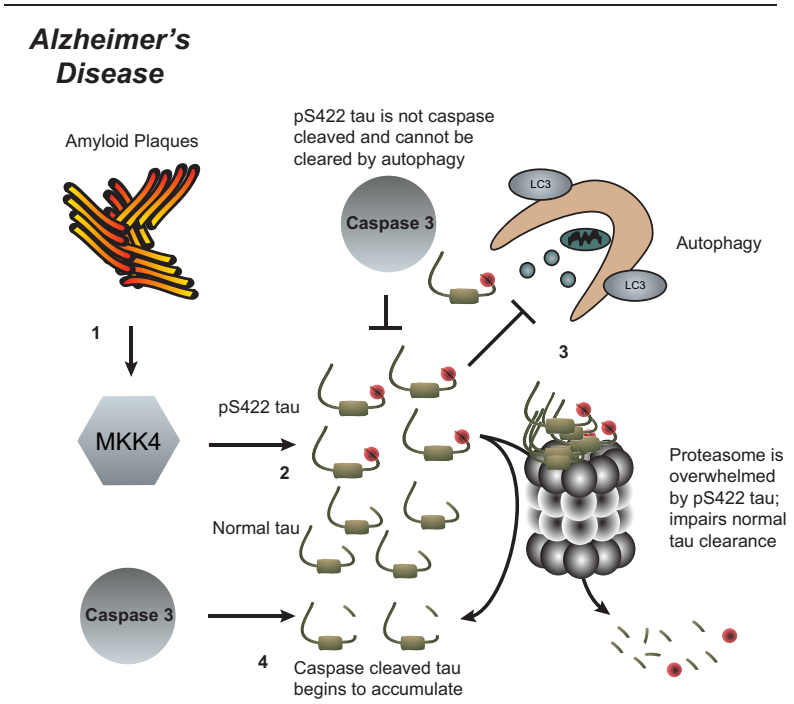

Figure 1. Proposed mechanism to explain data found by Vana et al. ${ }^{7}$ Under normal conditions, tau undergoes degradation by the proteasome or can be cleaved by caspase 3 and cleared via autophagy. In AD: 1) the presence of A $\beta$ plaques activates MAPK kinase 4, which 2) phosphorylates tau at S422, ${ }^{12,15,17}$ preventing caspase 3 from proteolytically cleaving tau at aspartic acid $421 .{ }^{8}$ 3) This pool of pS422 increases in size and cannot be cleared by autophagy, possibly actively inhibiting autophagy. The increasing amount of pS422 tau overwhelms the proteasome and leads to accumulation of soluble tau because the two major pathways of clearance are impaired. 4) Caspase 3 can cleave this new pool of soluble tau at D421, but because the two major clearance pathways are blocked, $\mathrm{TauC}^{+}$immunoreactivity is increased.

caspases and another that is not phosphorylated at S422 but still cannot be cleared because of inadequacy of the clearance machinery. This sequence of events would explain the observation of $\mathrm{TauC}^{+}$aggregates that appear after the initial appearance of pS422 tau (Figure 1). In this way, the findings presented herein provide extremely compelling rationale for further mechanistic studies focused on the phosphorylation of tau at $\mathbf{S 4 2 2}$ and its role in the initiation of tau pathogenesis.

The diversity of tau aggregate species, from dimers to NFTs, and the inability to precisely control oligomer size make it extremely challenging to identify which species of tau is toxic to neurons. ${ }^{26}$ The CBF human patient data from Vana et $\mathrm{al}^{7}$ show that pretangle tau species that occur early in disease progression and correlate with cognitive decline are more predictive of toxicity than NFT formation. These data corroborate previous studies using transgenic mouse models to elucidate the toxic species of tau and suggest that the oligomeric or pretangle tau aggregates are more toxic than NFTs. For example, a triple-transgenic $(3 \times \mathrm{Tg})$ mouse model (PS1 ${ }_{\mathrm{m} 146 \mathrm{v}}$, 
$\mathrm{APP}_{\text {swe }}$, and tauP301L) showed long-term potentiation deficits in an age-dependent manner, before either $A \beta$ or tau pathological characteristics. ${ }^{27}$ The clearance of tau by injection of $A \beta$ antibody in $3 \times \mathrm{Tg}$ mice reduced $A \beta$ and cleared early nonhyperphosphorylated tau but failed to clear later-stage hyperphosphorylated tau. ${ }^{28}$ When soluble amounts of $A \beta$ and tau were reduced together (not $A \beta$ alone), $3 \times \operatorname{Tg}$ mice with both pathological conditions recovered cognitive deficits, indicating that soluble amounts of tau affected the cognition of the mice. ${ }^{29}$ In another model, regulable (r)Tg4510 mice exhibit tetracycline-controlled expression of mutant P301L tau and NFT formation in neurons leads to neuronal loss and cognitive impairment. On suppression of tau expression, cognition was recovered, but NFT formation continued. ${ }^{30}$ In a region-specific manner, neuronal loss was evident before NFT formation in the dentate gyrus, a region where tau pathological features originate. ${ }^{31}$ Also, tau multimers of 140 and $170 \mathrm{kDa}$ that correlated with memory loss accumulated early in rTg4510 mice. These same multimers were found in a second tau transgenic line and in $A D$ and FTDP-17 human brain lysates. ${ }^{32}$

\section{Soluble pS422 Tau as the Earliest Marker for Disease}

Another example of the contribution of soluble tau species to neurotoxicity was shown in rTg4510 mice treated with the phenothiazine, methylene blue (MB). Although all tested doses of $\mathrm{MB}$ were neuroprotective, only those mice receiving doses of MB in excess of approximately $400 \mathrm{mg} / \mathrm{kg}$ showed cognitive improvement. This cognitive improvement was inversely correlated with soluble tau reductions; however, NFT formation was completely unaffected by MB treatment. ${ }^{33}$ Thus, reducing neither NFT load nor neuronal loss was sufficient to improve cognition; only when neuroprotection was accompanied by reduced soluble tau levels were cognitive deficits reversed. Thus, identifying pS422 tau as the earliest soluble tau species, as indicated by Vana et $\mathrm{al}^{7}{ }^{7}$ may be a first step toward designing effective taubased therapies that can halt tau pathogenesis and improve cognitive function.

\section{The Importance of Additional Markers: TauC3 Reactivity and Absence of $p 75^{N T R}$}

The progression of NFT formation in the medial temporal lobe neurons progressed such that the number of

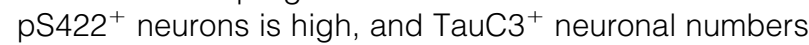
are low, but this ratio equalizes as the disease worsens. ${ }^{8}$ Herein, Vana et $\mathrm{al}^{7}$ show that, in CBF neurons, the ratio of

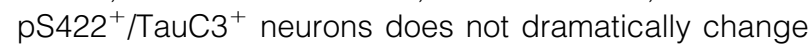
between patients with no cognitive impairment and patients with AD. This is interesting because deposition of tau into NFTs can occur at different rates, depending on the environment in which NFT formation is occurring. This idea has been previously proposed: changes to the local environment of in vitro tau reactions can dramatically change the way tau assembles into filaments. ${ }^{34,35}$ Vana et $\mathrm{al}^{7}$ also showed an increase in $\mathrm{TauC}^{+}$neurons, a decrease in $\mathrm{p} 75^{\mathrm{NTR}+}$ neurons, and a lack of $\mathrm{TauC}^{+} /$ p $75^{\mathrm{NTR}+}$ CBF neurons with the progression of $A D$. Caspase-mediated cleavage of tau is predictive of NFT formation, preceding even hyperphosphorylation. ${ }^{24,36,37}$ Because $\mathrm{NFT}^{+}$neurons are correlated with Braak staging of $\mathrm{AD}$ and $\mathrm{TauC}^{+}$reactivity predicts NFT formation, the presence of $\mathrm{TauC3}$ reactivity and the absence of p $75^{\mathrm{NTR}+}$ in these CBF neurons could represent a good marker for neurons that are on the cusp of degeneration. Combined with pS422 tau, this may be an excellent way to track the earliest stages of $A D$.

\section{Improving Early Diagnosis Is Essential for Effective Intervention}

$A D$ research is limited by an inability to properly resolve indicators of disease onset. Although our ability to diagnose prodromal $A D$ has certainly improved during the past several decades, it still is not possible to predict $A D$ with $100 \%$ accuracy. This limitation has greatly weakened the chances for clinical trial success. The samples used in this study highlight how poor our ability to resolve patient "sameness" is. The postmortem brain samples from various patients were grouped based on current standards of pathological and clinical diagnoses, and the results from the study show just how different these seemingly similar patients are. Perhaps using tools, such as those described herein, will improve the resolution for prodromal diagnosis, assist in postmortem analyses to reduce variability, and allow for a finer level of disease staging than the current mild cognitive impairment and five Braak stages.

Another possible utility for these findings is in the area of noninvasive imaging. Much work has been devoted to noninvasive tracking of $A D$ pathological characteristics. These types of studies provide a mechanism for longitudinal analyses of $A D$ pathological features in individual patients. By using labeled tracers, such as Pittsburgh compound $\mathrm{B}$, in conjunction with positron emission tomographic scanning, amyloid plaque deposition can be tracked in patients who develop AD. ${ }^{38}$ However, few tau tracers exist to be used in conjunction with Pittsburgh compound $\mathrm{B}$ and related probes. Recently, the compound ${ }^{18} \mathrm{~F}$-Thk523 was developed to track the formation of NFTs; unlike Pittsburgh compound B and other amyloid tracers, this tracer shows specificity for NFTs compared with $A \beta$ plaques. ${ }^{39}$ The insights provided by Vana et $\mathrm{al}^{7}$ may allow for the identification of a more specific tau epitope-specific tracer, such as one that recognized pS422, that could show pretangle tau pathogenesis. As we develop better technology, a beneficial compound that recognizes specific tau phosphorylation states or conformations would allow researchers to monitor these earlier changes.

In conclusion, Vana et $\mathrm{al}^{7}$ show that pretangle events in $\mathrm{CBF}$ neurons are associated with declines in cognitive functions. This is in opposition to NFT formation being the correlative factor. Future work investigating other changes to tau that cause the CBF neurons to degenerate would provide further insight into the mechanism of how tau causes 
toxicity. The results showing that NFT formation progresses at different rates, depending on the location of the neurons, are also of significance, suggesting that the microenvironment within discreet brain regions could be essential for disease susceptibility. Phosphorylation of tau at S422 within the CBF may be the earliest malfunction of a protein that is critical to the pathogenesis of many neurodegenerative diseases. These insights may lead to new therapeutic strategies targeting this toxic moiety.

\section{References}

1. Wilcock GK, Esiri MM: Plaques, tangles and dementia: a quantitative study. J Neurol Sci 1982, 56:343-356

2. Arriagada PV, Growdon JH, Hedley-Whyte ET, Hyman BT: Neurofibrillary tangles but not senile plaques parallel duration and severity of Alzheimer's disease. Neurology 1992, 42:631-639

3. Braak H, Braak E: Neuropathological stageing of Alzheimer-related changes. Acta Neuropathol 1991, 82:239-259

4. Sassin I, Schultz C, Thal DR, Rub U, Arai K, Braak E, Braak H: Evolution of Alzheimer's disease-related cytoskeletal changes in the basal nucleus of Meynert. Acta Neuropathol 2000, 100:259-269

5. Mesulam M, Shaw P, Mash D, Weintraub S: Cholinergic nucleus basalis tauopathy emerges early in the aging-MCI-AD continuum. Ann Neurol 2004, 55:815-828

6. Wu CK, Thal L, Pizzo D, Hansen L, Masliah E, Geula C: Apoptotic signals within the basal forebrain cholinergic neurons in Alzheimer's disease. Exp Neurol 2005, 195:484-496

7. Vana L, Kanaan NM, Ugwu IC, Wuu J, Mufson EJ, Binder LI. Progression of tau pathology in cholinergic basal forebrain neurons in $\mathrm{MCl}$ and AD. Am J Pathol 2011, 179:2535-2552

8. Guillozet-Bongaarts AL, Cahill ME, Cryns VL, Reynolds MR, Berry RW, Binder LI: Pseudophosphorylation of tau at serine 422 inhibits caspase cleavage: in vitro evidence and implications for tangle formation in vivo. J Neurochem 2006, 97:1005-1014

9. Guillozet-Bongaarts AL, Garcia-Sierra F, Reynolds MR, Horowitz PM, Fu Y, Wang T, Cahill ME, Bigio EH, Berry RW, Binder LI: Tau truncation during neurofibrillary tangle evolution in Alzheimer's disease. Neurobiol Aging 2005, 26:1015-1022

10. Guillozet-Bongaarts AL, Glajch KE, Libson EG, Cahill ME, Bigio E, Berry RW, Binder LI: Phosphorylation and cleavage of tau in non-AD tauopathies. Acta Neuropathol 2007, 113:513-520

11. Hefti F: Is Alzheimer disease caused by lack of nerve growth factor? Ann Neurol 1983, 13:109-110

12. Gotz J, Chen F, van Dorpe J, Nitsch RM: Formation of neurofibrillary tangles in P3011 tau transgenic mice induced by $\mathrm{A} \beta 42$ fibrils. Science 2001, 293:1491-1495

13. Gotz J, Chen F, Barmettler R, Nitsch RM: Tau filament formation in transgenic mice expressing P301L tau. J Biol Chem 2001, 276:529-534

14. Deters N, Ittner LM, Gotz J: Divergent phosphorylation pattern of tau in P301L tau transgenic mice. Eur J Neurosci 2008, 28:137-147

15. Grueninger F, Bohrmann B, Czech C, Ballard TM, Frey JR, Weidensteiner C, von Kienlin M, Ozmen L: Phosphorylation of Tau at S422 is enhanced by $A \beta$ in TauPS2APP triple transgenic mice. Neurobiol Dis 2010, 37:294-306

16. Ferrari A, Hoerndli F, Baechi T, Nitsch RM, Gotz J: $\beta$-Amyloid induces paired helical filament-like tau filaments in tissue culture. J Biol Chem 2003, 278:40162-40168

17. Grueninger F, Bohrmann B, Christensen K, Graf M, Roth D, Czech C Novel screening cascade identifies MKK4 as key kinase regulating Tau phosphorylation at Ser422. Mol Cell Biochem 2011, [Epub ahead of press]

18. Sheng JG, Jones RA, Zhou XQ, McGinness JM, Van Eldik LJ, Mrak RE, Griffin WS: Interleukin-1 promotion of MAPK-p38 overexpression in experimental animals and in Alzheimer's disease: potential significance for tau protein phosphorylation. Neurochem Int 2001, 39:341-348

19. Feijoo C, Campbell DG, Jakes R, Goedert M, Cuenda A: Evidence that phosphorylation of the microtubule-associated protein Tau by SAPK4/p38delta at Thr50 promotes microtubule assembly. J Cell Sci 2005, 118:397-408
20. Yoshida H, Goedert M: Sequential phosphorylation of tau protein by cAMP-dependent protein kinase and SAPK4/p38delta or JNK2 in the presence of heparin generates the AT100 epitope. J Neurochem 2006, 99:154-164

21. Zhu X, Rottkamp CA, Boux H, Takeda A, Perry G, Smith MA: Activation of p38 kinase links tau phosphorylation, oxidative stress, and cell cycle-related events in Alzheimer disease. J Neuropathol Exp Neurol 2000, 59:880-888

22. Munoz L, Ammit AJ: Targeting p38 MAPK pathway for the treatment of Alzheimer's disease. Neuropharmacology 2010, 58:561-568

23. Moh C, Kubiak JZ, Bajic VP, Zhu X, Smith MA, Lee HG: Cell cycle deregulation in the neurons of Alzheimer's disease. Results Probl Cell Differ 2011, 53:565-576

24. de Calignon A, Fox LM, Pitstick R, Carlson GA, Bacskai BJ, SpiresJones TL, Hyman BT: Caspase activation precedes and leads to tangles. Nature 2010, 464:1201-1204

25. Dolan PJ, Johnson GV: A caspase cleaved form of tau is preferentially degraded through the autophagy pathway. J Biol Chem 2010, 285: 21978-21987

26. Rankin CA, Gamblin TC: Assessing the toxicity of tau aggregation. J Alzheimers Dis 2008, 14:411-416

27. Oddo S, Caccamo A, Shepherd JD, Murphy MP, Golde TE, Kayed R, Metherate R, Mattson MP, Akbari Y, LaFerla FM: Triple-transgenic model of Alzheimer's disease with plaques and tangles: intracellular Abeta and synaptic dysfunction. Neuron 2003, 39:409-421

28. Oddo S, Billings L, Kesslak JP, Cribbs DH, LaFerla FM: Abeta immunotherapy leads to clearance of early, but not late, hyperphosphorylated tau aggregates via the proteasome. Neuron 2004, 43:321-332

29. Oddo S, Vasilevko V, Caccamo A, Kitazawa M, Cribbs DH, LaFerla FM: Reduction of soluble Abeta and tau, but not soluble Abeta alone, ameliorates cognitive decline in transgenic mice with plaques and tangles. J Biol Chem 2006, 281:39413-39423

30. Santacruz K, Lewis J, Spires T, Paulson J, Kotilinek L, Ingelsson M, Guimaraes A, DeTure M, Ramsden M, McGowan E, Forster C, Yue M, Orne J, Janus C, Mariash A, Kuskowski M, Hyman B, Hutton M, Ashe $\mathrm{KH}$ : Tau suppression in a neurodegenerative mouse model improves memory function. Science 2005, 309:476-481

31. Spires TL, Orne JD, SantaCruz K, Pitstick R, Carlson GA, Ashe KH, Hyman BT: Region-specific dissociation of neuronal loss and neurofibrillary pathology in a mouse model of tauopathy. Am J Pathol 2006, 168:1598-1607

32. Berger Z, Roder H, Hanna A, Carlson A, Rangachari V, Yue M, Wszolek Z, Ashe K, Knight J, Dickson D, Andorfer C, Rosenberry TL, Lewis J, Hutton M, Janus C: Accumulation of pathological tau species and memory loss in a conditional model of tauopathy. J Neurosci 2007, 27:3650-3662

33. O'Leary JC 3rd, Li Q, Marinec P, Blair LJ, Congdon EE, Johnson AG, Jinwal UK, Koren J 3rd, Jones JR, Kraft C, Peters M, Abisambra JF, Duff KE, Weeber EJ, Gestwicki JE, Dickey CA: Phenothiazine-mediated rescue of cognition in tau transgenic mice requires neuroprotection and reduced soluble tau burden. Mol Neurodegener 2010, 5:45

34. Carlson SW, Branden M, Voss K, Sun Q, Rankin CA, Gamblin TC: A complex mechanism for inducer mediated tau polymerization. Biochemistry 2007, 46:8838-8849

35. Voss K, Gamblin TC: GSK-3 $\beta$ phosphorylation of functionally distinct tau isoforms has differential, but mild effects. Mol Neurodegener 2009, 4:1-12

36. Rissman RA, Poon WW, Blurton-Jones M, Oddo S, Torp R, Vitek MP, LaFerla FM, Rohn TT, Cotman CW: Caspase-cleavage of tau is an early event in Alzheimer disease tangle pathology. J Clin Invest 2004, 114:121-130

37. Spires-Jones TL, de Calignon A, Matsui T, Zehr C, Pitstick R, Wu HY, Osetek JD, Jones PB, Bacskai BJ, Feany MB, Carlson GA, Ashe KH, Lewis J, Hyman BT: In vivo imaging reveals dissociation between caspase activation and acute neuronal death in tangle-bearing neurons. J Neurosci 2008, 28:862-867

38. Herholz K, Ebmeier K: Clinical amyloid imaging in Alzheimer's disease. Lancet Neurol 2011, 10:667-670

39. Fodero-Tavoletti MT, Okamura N, Furumoto S, Mulligan RS, Connor AR, McLean CA, Cao D, Rigopoulos A, Cartwright GA, O'Keefe G, Gong S, Adlard PA, Barnham KJ, Rowe CC, Masters CL, Kudo Y, Cappai R, Yanai K, Villemagne VL: 18F-THK523: a novel in vivo tau imaging ligand for Alzheimer's disease. Brain 2011, 134:1089-1100 